\title{
Layered packing and two-dimensional magnetism in honeycomb-type mixed oxides
}

Stanislav Podchezertsev $^{1}$, Artem Korshunov ${ }^{1}$, Alexander Malyshev ${ }^{1}$, Alexander Kurbakov ${ }^{1}$

${ }_{1}^{1}$ Petersburg Nuclear Physics Institute, NRC Kurchatov Institute, Gatchina, Russian Federation E-mail: stpcz@mail.ru

Quasi-two-dimensional magnetism is one of the most enthralling topics of a modern solid state physics. Reduced dimension gives rise to plenty of new phenomena. One of the most intriguing case for $2 \mathrm{D}$ lattices is a hexagonal net of antiferromagnetically ordered spins. Minimal possible for 2D lattices coordination number $(z=3)$, frustrated interactions of nearest neighbors with second and third neighboring spins and also a quantum fluctuations leads to a large variety of a possible ground states [1]. Fine examples of realization for such structures are mixed honeycomb oxides. Such compounds are described with chemical formulas: A2M2TeO6 and A3M2XO6 where A - is alkali, $\mathrm{M}-3 \mathrm{~d}$-metal and $\mathrm{X}$ is for $\mathrm{Sb}$ or $\mathrm{Bi}$ cations. Crystal structure of these compounds is formed by alternating layers of $\mathrm{Te} / \mathrm{Sb} / \mathrm{Bi}$ and $\mathrm{M}$ oxygen octahedra forming a honeycomb ordering and alkali cations with different type of oxygen surrounding depending on a structural politype. So magnetic ground state in these compounds depend on the nature of cations, their spin, orbital and electronic states determined by the local environment and also by a type of superstructure ordering due to a complexity of in- and interlayer exchange interactions. Still no adequate description between crystal structure and magnetic properties for such compounds was established, so the aim of a present work was to investigate crystal and magnetic structures of Li3Ni2SbO6 and $\mathrm{Na} 2 \mathrm{Ni} 2 \mathrm{TeO} 6$ compounds. For Li3Ni2SbO6 synchrotron diffraction experiment revealed a peak splitting that finally allowed to identify true space group to be $\mathrm{C} 2 / \mathrm{m}$; additional diffuse scattering indicating stacking faults presence was also detected. Neutron diffraction pattern at RT for $\mathrm{Na2Ni2TeO6}$ revealed an anisotropic peak broadening indirectly specifying a sample to be a possible mixture of a $\mathrm{P} 6322$ and $\mathrm{P} 63 / \mathrm{mcm}$ politypes. Close to 900 values of $\mathrm{Ni}-\mathrm{O}-\mathrm{Ni}$ bond angles shows presence of weak ferromagnetic inlayer interactions according to Goodenough-Kanamori rules; distorted $\mathrm{O}-\mathrm{Ni}-\mathrm{O}$ bond angles indicate a trigonal crystal field presence at Ni sites. LT neutron powder diffraction revealed addition peaks associated with magnetic scattering appearing at temperatures below 15 and $27 \mathrm{~K}$ for Li3Ni2SbO6 (on the left pic.) and Na2Ni2TeO6 (on the right pic.) respectively. Magnetic structures for both compounds are determined to be a zig-zag ferromagnetic chains coupled antiferromagnetically in ab-plane. Propagation vectors are $\mathrm{k}=\left(\begin{array}{lll}1 / 2 & 1 / 2 & 0\end{array}\right)$ for Li3Ni2SbO6 and $\mathrm{k}=\left(\begin{array}{lll}1 / 2 & 0 & 0\end{array}\right)$ for $\mathrm{Na} 2 \mathrm{Ni} 2 \mathrm{TeO} 6$. For Li3Ni2SbO6 ferromagnetic coupling for chains from adjacent layers was found, revealing non-negligible interlayer interactions. With temperature decreasing Ni spins directed along c-axis, demonstrates a certain tilt aligning perpendicular to ab-plane at $\mathrm{T}=1.5 \mathrm{~K}$. For $\mathrm{Na} 2 \mathrm{Ni2} \mathrm{TeO} 6$ increased ionic radius of alkali cation leads to a suppression of interlayer interactions. Ferromagnetic chains are coupled antiferromagnetically, nevertheless magnetic moments exhibit an inclination indicating a presence of a small ferromagnetic component within interlayer interactions.

[1] Li, P. H. Y., Bishop, R. F., Farnell, D. J., \& Campbell, C. E. (2012). Physical Review B, 86(14), 144404.

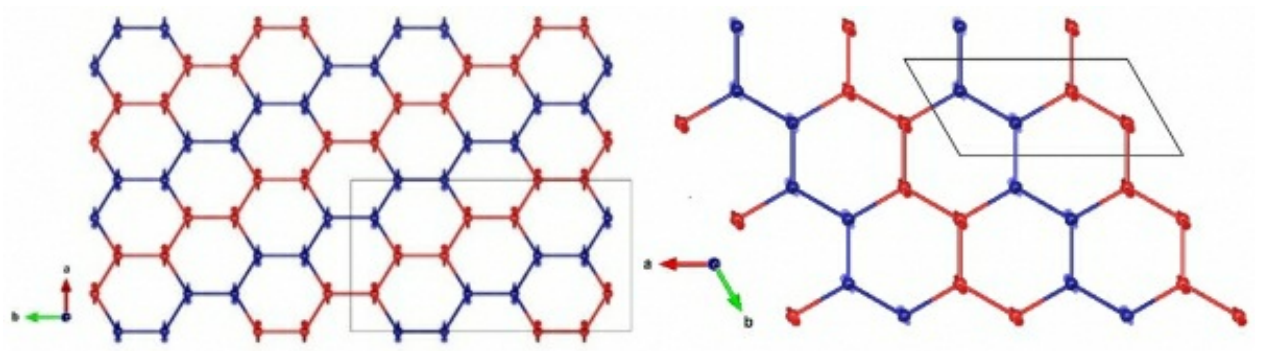

Keywords: magnetic structure, oxides, Rietveld refinement 